OPEN ACCESS

Citation: R. Vegliante, S. Miranda (2020) Costruire strumenti per lo sviluppo dell'intelligenza visuo-spaziale in età prescolare: il modello software VIEPapp. Media Education 11(2): 93-108. doi: 10.36253/me-9637

Received: August, 2020

Accepted: October, 2020

Published: December, 2020

Copyright: @2020R. Vegliante, S. Miranda. This is an open access, peerreviewed article published by Firenze University Press (http://www.fupress. $\mathrm{com} / \mathrm{me}$ ) and distributed under the terms of the Creative Commons Attribution License, which permits unrestricted use, distribution, and reproduction in any medium, provided the original author and source are credited.

Data Availability Statement: All relevant data are within the paper and its Supporting Information files.

Competing Interests: The Author(s) declare(s) no conflict of interest.

\section{Costruire strumenti per lo sviluppo dell'intelligenza visuo-spaziale in età prescolare: il modello software VIEP-apP ${ }^{1}$}

\author{
Creating tools for the development of visual-spatial intelligence \\ in preschool age: the VIEP-app software model
}

\author{
Rosa Vegliante, Sergio Miranda \\ Università di Salerno \\ E-mail: rvegliante@unisa.it; semiranda@unisa.it
}

\begin{abstract}
The health emergency caused by Covid-19 and the suspension of teaching activities in presence have required a total rethinking of the school system. Kindergarten has been most affected by the consequences of an exclusively remote system. The purpose of this paper is to present the theoretical-empirical premises of a playful-educational software model, the VIEP-app, a user-friendly application, aimed at increasing visual-spatial skills through a cognitive enhancement program that could be used in distance learning experiences. From a methodological point of view, this paper reports the experimental evidence relating to the connection between visual-spatial intelligence and the preschool period, using first and second level studies (systematic reviews or meta-analyzes). The review process made it possible to aggregate the different sources into three thematic areas: (i) the components of working memory; (ii) the exercise on executive functions in visual-spatial tests; (iii) the didactic effectiveness of the cognitive enhancement programs. The paper also concludes with some summary considerations on the possible research trends and their advancements are presented.
\end{abstract}

Keywords: empirical evidence, preschool age, visual-spatial skills, cognitive enhancement, software model.

Riassunto. L'emergenza sanitaria causata dal Covid-19 e la sospensione delle attività didattiche in presenza hanno imposto un totale ripensamento dell'assetto scolastico. La scuola dell'infanzia ha risentito maggiormente delle conseguenze scaturite da un sistema esclusivamente a distanza. Il contributo si pone l'obiettivo di presentare le premesse teorico-empiriche di un modello software ludico-educativo, denominato VIEP-app, un'applicazione user-friendly, volta a incrementare le abilità visuo-spaziali attraverso un programma di potenziamento cognitivo che possa essere utilizzato a distanza. Dal punto di vista metodologico, il lavoro riporta le evidenze sperimentali relative al nesso intelligenza visuo-spaziale e periodo prescolare avvalendosi di studi di primo e di secondo livello (revisioni sistematiche o meta-analisi). Il processo di revisione ha per-

\footnotetext{
${ }^{1}$ La stesura dell'articolo è frutto del lavoro congiunto degli autori. Rosa Vegliante ha redatto il paragrafo 2, Sergio Miranda ha redatto i paragrafi 1, 3 e 4.
} 
messo di aggregare le diverse fonti in tre aree tematiche: (i) le componenti della memoria di lavoro; (ii) l'esercizio delle funzioni esecutive nelle prove visuo-spaziali; (iii) l'efficacia didattica dei programmi di potenziamento cognitivo. Il contributo si conclude, inoltre, con alcune considerazioni di sintesi sulle possibili linee di ricerca e i relativi avanzamenti.

Parole chiave: evidenze empiriche, età prescolare, abilità visuo-spaziali, potenziamento cognitivo, modello software.

\section{INTRODUZIONE}

L'emergenza sanitaria, causata dal Covid-19, e le difficoltà ad essa connesse hanno reso necessario attivarsi per affrontare l'instabilità e l'incertezza derivate da un sistema scolastico esclusivamente a distanza. Il periodo di lockdown è servito innanzitutto per ripensare l'azione didattica e la relazione educativa sfatando, in particolar modo, quell'insieme di credenze ingenue sugli effetti negativi derivati dall'esposizione dei bambini ai media.

Ripensare con l'intenzione di ri-progettare le pratiche didattiche significa riorganizzare le modalità e i tempi per garantire quella continuità formativa nella quale i diversi attori (alunni, genitori e docenti) intrecciano contesti formali, non formali e informali (Amadini et al., 2019).

La nostra riflessione si è focalizzata su tre fattori: il bambino, i contenuti e il contesto (Lauricella et al., 2017) in una fase particolarmente delicata che è quella prescolare.

La scuola dell'infanzia, nello specifico, ha risentito maggiormente delle conseguenze derivate dalla sospensione della didattica in presenza, legate sia alla tenera età degli allievi che alla predisposizione di attività basate principalmente sulla relazione e sulla pratica esplorativa in un setting formativo accogliente. Al di là delle possibili soluzioni ipotizzate nel caso di un ritorno epidemico (MIUR, 2020), sarà inevitabile effettuare scelte metodologico-didattiche tese a favorire l'apprendimento tenendo conto sia dei bisogni formativi degli studenti che del valido supporto delle famiglie.

Significativo, in tal senso, è il contributo derivato dall'approccio Evidence based Education (EBE) (Calvani, 2007; 2012; Ranieri, 2007) che giustifica l'utilizzo delle strategie didattiche più efficaci da adottare in specifiche condizioni. Alcune evidenze empiriche (Clark et al., 2006; Clark, 2010) riportano la rilevanza di percorsi formativi nei quali gli stimoli vengano proposti in maniera graduale, $i$ compiti organizzati in sequenze, rispettando $i$ tempi di assimilazione, recupero e rinforzo dello studente rispetto alle informazioni presentate.

Centrale è dunque la mediazione didattica e, indipendentemente dallo spazio fisico, il bambino apprende esplorando in maniera attiva e costruttiva; agendo fisicamente; elaborando delle rappresentazioni o costruendo immagini mentali (Trinchero, 2017).
A partire dalla scuola dell'infanzia, la normativa italiana ribadisce l'importanza della pianificazione preventiva di attività didattiche, volte all'acquisizione delle competenze di base di natura cognitiva, emotiva e sociale (Gazzetta ufficiale, 2010; MIUR,1991; MIUR, 2012; MIUR, 2018). In questa specifica fase dello sviluppo evolutivo, il bambino è chiamato ad esperire una varietà di elementi, tratti dall'ambiente di apprendimento, così da facilitare il passaggio alla scuola primaria.

La prontezza scolastica, definita anche school readiness, insieme all'alfabetizzazione emergente (emergent literacy), si concretizzano mediante l'attuazione di processi formativi di pre-scolarizzazione basati sul potenziamento di funzioni cognitive, percettive e motorie. La school readiness, nello specifico, è un costrutto che abbraccia differenti prospettive e si pone come anello di congiunzione tra l'esercizio dei prerequisiti, ossia l'insieme di conoscenze, abilità e competenze necessarie per l'apprendimento successivo (Gagné, 1975) e la predisposizione di interventi compensativi qualora si manifestino delle difficoltà (Coggi \& Ricchiardi, 2014).

L'essere pronti per la scuola significa aver raggiunto quel livello di maturità legato all'età cronologica ma anche possedere quell'insieme di abilità utili ad apprendere i contenuti scolastici in un'ottica costruttiva e interattiva.

La letteratura internazionale (National Early Literacy Panel, 2008; NAEYC 2009) sottolinea la necessità di esercitare alcuni prerequisiti specifici, quali la discriminazione visiva e uditiva, la consapevolezza fonologica, la coordinazione oculo manuale, l'associazione visivo-verbale e la memoria fonologica, alla base delle competenze alfabetiche o matematiche.

Per ognuno di essi la meta analisi di Kaldenberg et al. (2015) propone una serie di attività di stimolazione da predisporre mediante l'attuazione di strategie didattiche, sistematicamente strutturate, nelle quali è centrale il ruolo del docente nel sollecitare la partecipazione dei discenti. A tal proposito, vengono presentati esercizi come la reiterazione e la ripetizione per consolidare la memoria e le funzioni esecutive; l'appaiamento o l'evidenziazione percettiva per affinare la discriminazione visiva e la corrispondenza biunivoca tra numero e parole per migliorare le abilità di calcolo. Nel contesto nazionale, Isidori e Prosperi (2019) hanno tratto dal lavoro di Kaldenberg et al. (2015) un approccio ludiforme per attuare un percor- 
so di sviluppo delle abilità di base con bambini di scuola dell'infanzia. Nell'esperienza descritta dalle autrici, la promozione dei precursori della competenza di pre-alfabetizzazione e pre-matematica ha consentito di effettuare uno screening precoce per risalire alle difficoltà emergenti. In tal modo, possono essere gettate le basi per favorire un solido e corretto apprendimento del codice numerico o linguistico con l'ingresso alla scuola primaria.

La nostra attenzione si è rivolta, in particolare, alle abilità non verbali e visuo-spaziali che consentono al bambino di affinare la percezione visiva per agire nello spazio e intervenire sulle rappresentazioni mentali, mediante funzioni di orientamento (Baddeley \& Hitch, 1974; Cornoldi \& Vecchi, 2003; Gardner, 1983). Da qui deriva, oltretutto, la necessità di sviluppare quel potenziale di abilità nei differenti domini cognitivi e percettivo-motori. Nell'ambito di questo considerevole interesse per lo studio dei prerequisiti richiamati, si ritiene che la memoria di lavoro assuma una posizione centrale nel moderare il funzionamento delle abilità di base a partire dall'acquisizione della consapevolezza fonologica o della competenza numerica (Brizzolara et al., 1999; Bull et al. 2008; Gathercole \& Baddeley, 1989; Gathercole \& Alloway, 2008; Oakhill \& Kyle, 2000; Orsolini et al., 2003; Siegler \& Shrager, 1984).

L'obiettivo degli autori è di presentare, partendo da queste premesse, la letteratura a sostegno dell'impianto progettuale del modello VIEP-app, un'applicazione userfriendly per computer, tablet e smartphone, destinata ai bambini in età prescolare e sviluppata nell'ambito del progetto di ricerca Visual Intelligence Enhancement Program ${ }^{2}$ (VIEP) (Calvani \& Zanaboni, 2018). In questo momento storico, proprio l'applicazione e quindi il modello attraverso il quale è stata progettata assume un aspetto differente poiché, grazie all'uso dei dispositivi tecnologici citati, meglio se dotati di schermo tattile, consentirebbe ai bambini di svolgere a distanza delle esperienze che sarebbero possibili quasi esclusivamente in presenza.

L'intento è di proporre considerazioni di sintesi sui possibili avanzamenti della ricerca dimostrando, al contempo, in quali situazioni e secondo quali modalità un software ludico-educativo, specificamente progettato, possa contribuire all'elaborazione delle rappresentazioni visuo-spaziali in età prescolare.

\section{OGGETTO D'ANALISI E APPROCCIO METODOLOGICO}

Nel ricercare le evidenze empiriche a sostegno dei programmi di potenziamento delle abilità di base, da

\footnotetext{
${ }^{2}$ Il progetto VIEP è stato realizzato con il supporto della Società per l'Apprendimento e l'Istruzione informati da Evidenza (S.Ap.I.E.).
}

attuare nella scuola dell'infanzia, ci si è avvalsi di studi di primo e di secondo livello (revisioni sistematiche o meta-analisi) tratti dai principali database elettronici (ERIC e Web of Science) e da Google Scholar.

Dal punto di vista metodologico, nel ricostruire il framework teorico, la scelta è ricaduta sull'elaborazione di una sintesi della letteratura mediante un approccio descrittivo e interpretativo (Fabbro, 2018). A differenza di una rassegna sistematica, tale modalità consente di re-interpretare gli studi selezionati con maggiore profondità, aggregandoli per analogia argomentativa o oppositiva al fine di effettuare delle possibili comparazioni. Per tale ragione, si riportano in dettaglio le fasi seguite nella conduzione dell'analisi. Il processo di revisione, da non confondersi con il meta-narrative mapping (Noblit \& Hare, 1988), ha previsto di: identificare il focus della sintesi; individuare gli studi più rilevanti; riassumere le questioni emergenti (partecipanti, metodi e strumenti utilizzati, risultati); evidenziare possibili relazioni tra gli studi; risalire a categorie di analisi; argomentare le differenti problematiche per disseminare i risultati.

Da una prima analisi delle fonti, inerente al nesso intelligenza visuo-spaziale e periodo prescolare, sono derivate le seguenti sotto-categorie tematiche: (i) le componenti della memoria di lavoro; (ii) l'esercizio delle funzioni esecutive nelle prove visuo-spaziali; (iii) l'efficacia didattica dei programmi di potenziamento cognitivo.

\subsection{Le componenti della memoria di lavoro}

Studi accreditati sostengono che il processamento visivo e l'attenzione visuo-spaziale giochino un ruolo fondamentale nell'apprendimento e nell'automatizzazione dei meccanismi sottesi alla letto-scrittura (Cornoldi et al. 1994; Fastame \& Antonini, 2011; Goff et al., 2005; Kellogg, 1996; Kellogg et al., 2013). Da ciò deriva la possibilità, da parte del bambino, di discriminare e riconoscere la corrispondenza tra le unità linguistiche minime (grafemi e fonemi). L'eventuale compromissione del sistema visivo rientra nel novero delle cause della dislessia, infatti la mancata incidenza del processamento visivo dell'informazione sulla capacità di lettura è riconducibile a differenti forme di disturbo visuo-spaziali (Lucchiari, 2018). Nella verifica dell'integrità visiva, l'optometria americana ricorre al modello pratico di analisi clinica, elaborato da Scheiman e Rouse (2006), nel quale il processamento dell'informazione, o meglio la sfera della percezione, rappresenta la terza area dell'acuità visiva insieme all'integrità e all'efficienza della visione. Secondo tale prospettiva, le abilità visuo-spaziali coincidono con azioni di identificazione e di discriminazione del contesto da integrare con gli altri sensi per accrescere la consapevolezza dello 
spazio. Nella macrocategoria dell'elaborazione visiva rientrano le seguenti componenti:

- le abilità visuo-spaziali, nelle quali si annoverano la lateralità, la direzionalità e l'integrazione bilaterale; - l'analisi visiva ossia il riconoscimento, la costanza di forme, misure e la chiusura visiva;

- la memoria visiva, la visualizzazione mentale e la velocità di percezione visiva;

- l'integrazione visuo-motoria e uditiva (Lucchiari, 2018; Scheiman \& Rouse, 2006).

In particolare, le abilità visuo-spaziali rappresentano quell'insieme di capacità che consentono al soggetto di affinare la percezione visiva per agire nello spazio e intervenire sulle rappresentazioni mentali, mediante funzioni di orientamento (Maffioletti \& Facchin, 2016). Lo spazio, infatti, ha una natura composita, è determinato da molteplici fattori tra cui la memoria di lavoro e le funzioni esecutive. Tali componenti si definiscono anche predittori delle abilità sottese all'apprendimento delle discipline scolastiche. La memoria di lavoro gestisce processi e meccanismi specializzati nel controllo, nel mantenimento e nella regolazione delle informazioni rilevanti (Miyake \& Shah, 1999). È un sistema articolato e strutturato in differenti codici e rappresentazioni multiple che si lega alla memoria a lungo termine. Inizialmente, si riteneva che la memoria di lavoro visuo-spaziale avesse delle analogie con la memoria verbale. Studi successivi hanno sempre più focalizzato l'attenzione su tali aspetti cercando di risalire a una possibile architettura.

Secondo il modello multi-componenziale (Baddeley \& Hitch, 1974; Baddeley, 1986), la memoria di lavoro è costituita da tre sistemi: una centrale esecutiva (central executive) che media e coordina l'area articolatoriofonologica (articulatory loop) e il taccuino visuo-spaziale (visuo-spatial sketchpad), specializzato a sua volta in due subsistemi deputati alla conservazione delle informazioni e all'elaborazione delle risorse visuo-spaziali. Il modello originale si arricchisce di un'ulteriore interfaccia, l'episodic buffer, che funge da componente intermedia tra i subsistemi verbale e visivo con il compito di produrre una rappresentazione integrata tratta dalle differenti informazioni (Baddeley, 2000; 2002).

Nel panorama internazionale e nazionale, l'interesse verso tale questione si è allargato rapidamente vedendo l'affermarsi di studi volti a comprendere le funzionalità della memoria di lavoro, in particolare le modalità esecutive del loop articolatorio e del taccuino visuo-spaziale nel processo di elaborazione degli stimoli sensoriali dei bambini.

Ricerche sperimentali provano, infatti, che in età infantile le due aree sopra indicate risultano separate sebbene il taccuino visuo-spaziale sia maggiormente connesso alle funzioni dell'esecutivo centrale (Gathercole \& Pickering, 2000; Pickering et al., 1998). Quanto affermato viene giustificato dal fatto che all'età di 3-4 anni i bambini si avvalgono del canale visivo per la codifica delle informazioni verbali che, successivamente, si trasforma in doppia codifica verbale e visiva (Vianello et al., 2015). Logie e collaboratori (Logie, 1986; Logie \& Marchetti, 1991) hanno ipotizzato la separazione interna al taccuino visuo-spaziale in specifiche subcomponenti cognitive: la visual cache, un magazzino di informazioni visive e l'inner scribe, un sistema temporaneo per il mantenimento del movimento o di sequenze di movimenti nello spazio. Attraverso tecniche, incentrate sull'interferenza selettiva, sono state associate prove visive (il ricordo di forme colorate) o spaziali (sequenze di movimenti) a compiti secondari concernenti stimoli irrilevanti di natura visiva e spaziale. I risultati ottenuti hanno confermato che sia le prove visive che quelle spaziali vengono eseguite da aree indipendenti, pur se contrastate da compiti interferenti della medesima tipologia.

Nel comprovare l'esistenza di una varietà di risorse coinvolte nell'elaborazione visuo-spaziale e la separazione tra le componenti di natura verbale e non, le ricerche di Cornoldi e Vecchi $(1998 ; 2000 ; 2003)$ hanno teorizzato un modello di memoria di lavoro (teoria dei Continua) paragonabile a una struttura unitaria di forma conica, caratterizzata da un continuum verticale e orizzontale. L'uno (continuum verticale) pone in relazione compiti attivi e passivi che richiedono un basso o elevato livello di elaborazione, l'altro (continuum orizzontale) invece la tipologia di materiale impiegato nel compito specifico e la possibile distinzione tra formato e modalità di presentazione. Nella teoria dei Continua, l'elaborazione delle informazioni dipende sia dal processo richiesto per la risoluzione del compito che dal suo formato, ossia dal dominio specifico previsto (verbale, visivo e spaziale). Ne deriva che le strutture interne alla memoria di lavoro si predispongono lungo un continuum i cui estremi sono contraddistinti da un lato dai processi di elaborazione/ integrazione e, dall'altro, da meccanismi passivi orientati all'archiviazione dei formati nei domini-specifici. Il ricordo passivo di questi ultimi non si avvale delle componenti centrali di elaborazione, a differenza dei processi rielaborativi che si indirizzano sempre più verso le componenti di tipo centrale. Pur tuttavia sia che si tratti delle modalità elaborativo-integrative sia della tipologia del compito, il posizionamento lungo il continuum centrale-periferico dipende anche dal carico attentivo, che «è maggiore quanto più il processo avviene a un livello centrale» (Vecchi \& Cornoldi, 1998, p. 504).

Le prestazioni legate alla rotazione mentale degli stimoli, alla generazione e trasformazione delle figure, 
così come al mantenimento e allo scanning di immagini, vengono svolte in maniera differente dai bambini di diversa età (Kosslyn et al., 1990). La memoria di lavoro visuo-spaziale è influenzata dallo sviluppo cognitivo, infatti la rotazione mentale risulta correlata anche alla lateralizzazione, ossia a quel processo di espressione della dominanza emisferica che termina con la consapevolezza di utilizzare correttamente i riferimenti spaziali "destra" e "sinistra" (Roberts \& Aman, 1993). I bambini in età prescolare riescono a svolgere compiti visivi e spaziali che richiedono un basso carico attentivo: più si familiarizza con determinati stimoli, più si registra un miglioramento delle prestazioni (Hitch et al., 1988). Se si considera il genere, le bambine si mostrano più abili in prove verbali o in compiti nei quali è richiesto il ricordo passivo volto a rintracciare i tratti visivi nelle immagini, rispetto ai bambini che manifestano una spiccata propensione in prestazioni visuo-spaziali, quali la rotazione e la trasformazione mentale (Clark \& Paivio, 1987; Maccoby \& Jacklin, 1974; Mckelvie, 1986; Vecchi \& Cornoldi, 1998).

Le ricerche condotte da Gathercole et al. (2004) hanno evidenziato come, con l'avanzare dell'età, le specifiche capacità delle tre componenti della memoria di lavoro vengano affinate sino all'adolescenza. All'iniziale separazione tra i domini specifici (verbale e visuo-spaziale) e i domini generali (controllo inibitorio e attentivo), durante la crescita si attivano delle connessioni tra i differenti magazzini (verbale e visivo) e il sistema centrale mediante lo sviluppo di specifiche strategie deputate al controllo, alla gestione e all'archiviazione delle risorse nella memoria a breve termine. Quanto affermato è stato dimostrato anche dalla ricerca condotta da Lanfranchi e Vianello (2009) nella quale sono state somministrate 3 prove di natura verbale e 3 di carattere visuo-spaziale con differenti livelli di controllo a 247 bambini di 4-6 anni. I risultati ottenuti mostrano come vi sia una correlazione significativamente superiore $(\mathrm{p}<.001)$ tra la prova ad alto controllo verbale e quella ad alto controllo visuo-spaziale. Le prove verbali a basso controllo non risultano correlate alle prove a medio e alto controllo visuo-spaziale. Di contro, la prova visuo-spaziale a basso controllo è correlata positivamente con quelle verbali a medio e alto controllo. Questo risultato fa ipotizzare che già a 4 anni la memoria di lavoro attivi processi di attenzione e controllo supportati dai magazzini verbale e visuo-spaziale. Quanto affermato viene provato anche da Alloway et al. (2006) i quali individuano una relazione significativa tra il magazzino visuo-spaziale e l'esecutivo centrale rispetto alla memoria verbale, che agisce in maniera separata dai domini generali. Ricerche neuroanatomiche mostrano che il sistema visivo negli esseri umani è composto da due vie anatomico-funzionali e che l'elaborazione degli stimoli venga gestita da differenti circuiti afferenti alla memoria di lavoro verbale, spaziale e visiva (Mammarella, 2008). La possibilità di richiamare gli oggetti e di individuare la loro posizione nello spazio, attività specifiche della memoria spaziale, contribuiscono a rafforzare il processo di lateralizzazione a destra mentre la memoria visiva e verbale sono implicate nella lateralizzazione a sinistra con una peculiare attivazione delle aree cerebrali (regione occipitale e infero-frontali per la memoria spaziale) e infero-temporali per il ricordo visivo. Tale dissociazione tra area visiva e spaziale si esplica nelle differenti modalità di codifica: extrafigurale, subentra nel momento in cui ci si trova dinanzi a stimoli esterni; intrafigurale, consente di rintracciare la relazione tra le posizioni interne alle figure e una terza codifica che scompone quella intrafigurale nella possibilità di stabilire delle relazioni tra i percorsi o tra le configurazioni. Un'ulteriore distinzione risiede tra spazialità sequenziale, richiesta per la risoluzione del test di Corsi ${ }^{3}$ e spazialità simultanea, necessaria per rievocare la posizione assunta dagli oggetti all'interno della matrice in contemporaneo a compiti visivi (De Ribaupierre et al., 2000; Lecerf \& De Ribaupierre, 2005; Pazzaglia, 1999). Dunque, prima dell'età della scolarizzazione, la codifica e il ricordo del materiale verbale sono appannaggio del dominio visivo semantico e non fonologico (Lanfranchi \& Vianello, 2009).

\subsection{Lesercizio delle funzioni esecutive nelle prove visuo-spa- ziali}

Nelle ricerche che affrontano l'intelligenza visiva emerge un comprovato legame tra le funzioni esecutive e le abilità visuo-spaziali, in particolare nel monitoraggio di immagini in sequenza e nelle operazioni di regolazione cognitiva (Duff \& Logie, 1999; Logie, 1995). I formati dei compiti visuo-spaziali implicano l'attivazione delle funzioni esecutive, ossia l'insieme di quei processi selettivi, attentivi e regolativi dei comportamenti implicati nella risoluzione di un problema (Diamond \& Lee, 2011; Diamond, 2013; Welsh \& Pennington, 1988).

L'efficacia didattica dei percorsi basati sulle funzioni esecutive rappresenta uno dei temi più frequenti della ricerca educativa riguardante la school readiness. I docenti di scuola dell'infanzia, infatti, riconoscono come prioritario lo sviluppo di competenze, quali l'auto-

\footnotetext{
${ }^{3}$ Il test di Corsi (1972) riprende il test del cubo di Knox (1913) per ciò che concerne l'utilizzo dei cubi e la sequenza ma varia il numero e lordine dei blocchi. È una batteria indicata per valutare le componenti della memoria di lavoro visiva e spaziale.
} 
controllo e l'attenzione in età prescolare rispetto all'acquisizione precoce dei contenuti disciplinari (RimmKaufman et al., 2000). La letteratura di riferimento riporta il coinvolgimento di tali funzioni in compiti che richiedono la pianificazione, la flessibilità, l'autoregolazione e la capacità di avviare un'azione (Anderson, 2002; Diamond et al., 2007; Pennington \& Ozonoff, 1996). Il rimando va a quel complesso di attività strettamente connesse alla memoria di lavoro, all'attenzione e al controllo inibitorio. Secondo il modello di Miyake et al. (2000) le funzioni esecutive si suddividono in tre domini tra loro connessi: l'inibizione (inhibition) di informazioni irrilevanti, l'aggiornamento (updating) delle informazioni nella memoria di lavoro verbale e visuo-spaziale e la flessibilità cognitiva (shifting).

Nel modello proposto da Diamond (2013) non solo vengono ripresi i tre domini di Miyake et al. (2001) ma viene enfatizzato il controllo inibitorio. Si avvalora l'incidenza della memoria di lavoro sull'autocontrollo, sull'inibizione cognitiva e sull'attenzione selettiva-focalizzata. La memoria di lavoro e il controllo inibitorio, a loro volta, condizionano la flessibilità cognitiva che agisce sulla pianificazione, sul ragionamento e sulla capacità di problem solving. Nella descrizione del modello, Diamond sottolinea lo stretto legame tra la memoria di lavoro e l'attenzione giustificando il fatto che i miglioramenti delle prestazioni, riguardanti l'attivazione della memoria di lavoro, si riscontrano anche in quelle inerenti al campo dell'attenzione.

Le analisi condotte con bambini di età scolare presentano una sostanziale differenziazione tra i domini delle funzioni esecutive rispetto alla fascia d'età prescolare (Wiebe et al., 2011). Nella prima infanzia, il controllo esecutivo risulterebbe un costrutto unitario difficilmente suddivisibile nelle sue componenti specifiche in quanto, anche dal punto di vista anatomico, le aree della corteccia cerebrale, deputate all'analisi visiva e uditiva, così come i sistemi dedicati ai compiti associativi, attivano una densità di connessioni sinaptiche che si riducono considerevolmente con l'avanzare dell'età (Anderson, 2012). In questa fascia d'età, il controllo inibitorio subentra in quelle prove che presuppongono maggiore attenzione, permettendo così di spiegare la differenza tra le prestazioni dei bambini.

Intorno ai 4 anni, si assiste a una separazione tra il controllo inibitorio e la memoria di lavoro. Solo successivamente, dinanzi a compiti complessi, i bambini associano il controllo inibitorio alla memoria di lavoro. Quest'ultima si affina proprio nella seconda metà del periodo prescolare con lo sviluppo dell'attenzione (Garon et al., 2008). Dagli 8 anni in poi le funzioni esecutive permettono di regolare il funzionamento cogni- tivo coinvolgendo, in tal senso, la memoria di lavoro, l'inibizione e la flessibilità. Tuttavia la sinergia tra l'attenzione selettiva, la memoria verbale, visuo-spaziale e la flessibilità cognitiva sembrerebbero determinanti nel processo di apprendimento futuro del bambino. Si tratta di elementi implicati in tutte quelle esperienze didattiche che richiedono l'esercizio del linguaggio o della memoria. Dinanzi alla risoluzione di problemi, la memoria di lavoro consente di elaborare una rappresentazione globale del testo e attivare delle connessioni con le informazioni preesistenti, al contempo il controllo inibitorio interviene per ostacolare l'utilizzo di dati o di rappresentazioni non idonee. Lo studio condotto da Bull e Scerif (2001) riporta, tra le principali difficoltà in matematica, la scarsa funzionalità della memoria di lavoro e la mancanza del controllo inibitorio registrando ripercussioni notevoli nell'applicazione di strategie d'analisi per fronteggiare il compito. Ciò dimostra quanto sia importante in età prescolare potenziare la memoria di lavoro e il controllo inibitorio per affinare quell'insieme di abilità sottese alla competenza matematica (Espy et al., 2004). In questo periodo dello sviluppo, infatti, la capacità inibitoria, il controllo attentivo e l'attivazione di strategie, volte a pianificare azioni finalizzate, registrano un significativo incremento (Brocki et al., 2007; Davidson et al., 2006). Dall'incisività della memoria di lavoro nel mantenere provvisoriamente le informazioni sugli stimoli di posizione e dalle abilità visuo-costruttive dipendono le abilità di conteggio (Kyttälä et al., 2003). Inoltre, le prove spaziali predicono le prestazioni matematiche in bambini di 7-8 anni, mentre quelle visive in bambini di 9-10 anni (Holmes et al., 2008). Ne deriva che le difficoltà nell'apprendimento matematico sono causate da un deficit della memoria di lavoro in particolare dall'inefficienza dell'esecutivo centrale e dal controllo inibitorio (Passolunghi \& Siegel, 2004). Quanto affermato viene confermato dalla correlazione esistente tra il deficit della memoria spaziale e le scarse prestazioni in compiti di calcolo che, ad esempio, richiedono di incolonnare i numeri o di distinguere i segni algebrici (McLean \& Hitch, 1999; Rourke, 1993). Dunque, bambini poco abili nella risoluzione dei problemi riportano prestazioni peggiori in prove spaziali rispetto a quelle visive o verbali (Cornoldi et al., 1999; Passolunghi \& Mammarella, 2010).

Passolunghi et al. (2011) hanno verificato la relazione esistente tra difficoltà in matematica e deficit in abilità visuo-spaziali. In un campione di 321 alunni di classi terze, quarte e quinte della scuola primaria, sono stati estratti 81 alunni suddividendoli in due gruppi: uno composto da 40 bambini abili e l'altro composto da 41 bambini che riscontrano difficoltà nell'apprendi- 
mento della matematica e nella risoluzione dei problemi. Ad essi sono state somministrate prove inerenti alla memoria di lavoro visiva, spaziale e verbale concernenti il semplice mantenimento in memoria o l'elaborazione delle informazioni. I bambini meno abili hanno registrato prestazioni inferiori nei compiti di natura spaziale rispetto a quelli visivi e verbali. Gli esiti confermano la rilevanza della memoria spaziale nella capacità risolutiva in compiti che mantengono la loro specificità indipendentemente dal livello di elaborazione richiesto o dal controllo attentivo.

L'incidenza della memoria di lavoro nella risoluzione dei problemi viene ricondotta all'incapacità di elaborare, inibire e trattenere le informazioni pertinenti (Passolunghi, 2004). In tale operazione è coinvolto il magazzino visuo-spaziale che trattiene temporaneamente l'informazione ed è rilevante nella generazione e nella trasformazione della rappresentazione mentale. Dalla visualizzazione si passa all'analisi descrittivo-analitica delle figure. Come riporta Del Grande (1990), nella cognizione geometrica, ad esempio, le abilità spaziali gestiscono la coordinazione oculomotoria, l'individuazione delle figure sullo sfondo, la percezione della costanza della forma e della posizione nello spazio, la discriminazione visiva e la memoria visiva. I percorsi di potenziamento realizzati da Freina e Bottino (2019), in classi quarte e quinte di scuola primaria, confermano la rilevanza di tali abilità sul miglioramento degli apprendimenti scolastici. Nelle classi sperimentali è stato implementato un percorso di stimolazione che ha previsto l'adozione di giochi digitali riguardanti l'esercizio delle abilità predette. $\mathrm{Al}$ termine della sperimentazione, i risultati ottenuti nelle prove di matematica comprovano il miglioramento delle prestazioni, dato che si allinea anche con gli esiti conseguiti, dalle stesse classi, nelle prove Invalsi.

\subsection{Lefficacia dei programmi di potenziamento cognitivo}

L'esercizio sistematico e precoce delle capacità cognitive di base, ossia la percezione visiva, il problem solving, la memoria di lavoro, le abilità visuo-spaziali e la cognizione numerica, consente di apportare delle modifiche alle componenti delle funzioni esecutive al fine di compensare eventuali abilità assenti o compromesse. Il potenziamento cognitivo, infatti, mira ad ampliare tali capacità in modalità graduale e controllata così da favorire una sorta di allenamento mentale atto a fronteggiare compiti nuovi e sfidanti, avvalendosi di strategie efficaci (Trinchero, 2009; 2014).

La revisione sistematica, condotta da Anderson et al. (2003) sull'efficacia dei programmi di sviluppo da attuare nella prima infanzia, prova l'effetto positivo nel prevenire eventuali ritardi nello sviluppo cognitivo e nell'abbandono scolastico. Ciò viene confermato dagli esiti riportati da test standardizzati, quali ad esempio la batteria Wechsler Preschool and Primary Scale of Intelligence - Fourth Edition (Wechsler, 2012), volta a misurare la prontezza scolastica o il TPV-Percezione visiva e integrazione visuo-motoria che rileva, in maniera preventiva, la capacità di integrazione visuo-motoria e le abilità percettivo-visive (Hammill et al., 2003). Per contrastare eventuali difficoltà di lettura, scrittura o calcolo, che potrebbero insorgere nel periodo della scolarizzazione, sono stati messi a punto diversi strumenti standardizzati con lo scopo di misurare le cosiddette abilità di school readiness. La rassegna di Maniscalco et al. (2015) riporta i principali test psicometrici utilizzati per verificare le abilità di base tali da consentire uno screening precoce prima dell'ingresso nella scuola primaria. Tra questi si annoverano il questionario di Identificazione Precoce delle Difficoltà di Apprendimento IPDA (Terreni et al., 2011), il Test di valutazione delle Competenze Meta-Fonologiche CMF (Marotta et al., 2008), la batteria di School Readiness SR 4-5 (Zanetti \& Miazza, 2003), le Prove di Abilità Cognitive per la scuola dell'infanzia PAC-SI (Scalisi et al., 2009) e lo Screening Prescolare Età Evolutiva Dislessia SPEED (Savelli et al., 2013). A tal proposito, il vantaggio di pianificare interventi mirati viene dimostrato da studi di follow-up che, anche a distanza di un anno, accertano i progressi delle prestazioni di bambini rispetto alle carenze iniziali (Coggi \& Ricchiardi, 2014).

A qualsiasi età le funzioni esecutive possono essere promosse e incrementate con modalità e approcci differenti in tempi brevi o lunghi. La stessa fluidità cognitiva, parte integrante del ragionamento e determinante nel processo risolutivo dei problemi, può essere migliorata mediante un training formativo basato sull'esercizio ripetuto (Diamond, 2013).

La rappresentazione e la riproduzione dei modelli visivi e spaziali, così come la percezione delle forme, se misurate all'ultimo anno della scuola dell'infanzia, predicono i risultati futuri in matematica (Zhang \& Lin, 2015). Quanto affermato avvalora la forte relazione tra le abilità spaziali e le conoscenze matematiche in età prescolare (Mix \& Cheng, 2012). Il pensiero matematico è supportato dalle rappresentazioni spaziali soprattutto nella risoluzione dei problemi che, alla scuola primaria, richiedono la combinazione di oggetti o la separazione di insiemi (addizione e sottrazione) (Caviola et al., 2012). L'esercizio delle capacità cognitive connesse alle immagini visive, alla manipolazione mentale delle informazioni spaziali o all'assemblaggio spaziale, diventano centrali nei processi di apprendimento sin dai primi anni d'i- 
struzione (Newcombe, 2010; Verdine et al., 2017). La letteratura di riferimento riporta risultati incoraggianti in merito, anche se tratti da prospettive differenti. Alcuni studi longitudinali confermano la causalità tra il miglioramento delle abilità visuo-spaziali nell'infanzia e il successivo progresso in prove di matematica (Hawes et al., 2017; Lowrie et al., 2017). Altri si soffermano sull'analisi di specifiche componenti alla base della competenza numerica, quali il conteggio o la rappresentazione spaziale dei numeri mediante la linea numerica mentale, riconoscendo l'incidenza di fattori culturali ed educativi al di là di quelli genetici (Bruandet et al., 2004; Dehaene et al., 2003). La sperimentazione di Xu e LeFevre (2016), condotta con 83 bambini dai 3 ai 5 anni, a seguito di una sessione di training basata sull'esercizio di attività legate alla decomposizione spaziale delle forme, non registra risultati significativi in compiti di ordinamento numerico rispetto agli esiti positivi ottenuti dopo sessioni di allenamento sequenziale (incentrate ad esempio su cosa viene prima o dopo un dato numero). Il principio di cardinalità numerica non implica il possesso di una coerente rappresentazione spaziale del numero, quest'ultima invece si correla alla capacità di operare dei confronti tra grandezze numeriche, ciò significa che i bambini con una buona mappatura spaziale ottengono prestazioni migliori in compiti che prevedono la selezione della cifra più grande tra quelle visibili (Le Corre, 2014; Sella et al., 2017).

Di contro, l'inefficienza della memoria di lavoro visuo-spaziale ha importanti ripercussioni non solo in specifiche attività visive e spaziali ma anche in procedure di calcolo, dovute principalmente all'incapacità di rappresentare mentalmente i numeri (Mammarella et al., 2010; Rotzer et al., 2009).

Il miglioramento delle funzioni esecutive, in età prescolare, viene garantito soprattutto se preceduto dall'implementazione di un programma formativo rivolto ai docenti (Tominey \& McClelland, 2011), annoverabile nell'ambito della ricerca-formazione. Lo studio di Walk et al. (2018) restituisce l'efficacia di un programma d'intervento (EMIL), realizzato in quattro scuole dell'infanzia tedesche, che ha visto il coinvolgimento attivo dei docenti nel mettere in pratica i contenuti acquisiti durante un periodo di formazione. Il piano di ricerca ha previsto un gruppo sperimentale (72 bambini) e un gruppo di controllo (61 bambini) di età media pari a 48 mesi sottoposti a sette test in ingresso, intermedi e finali atti a verificare le funzioni esecutive. Al termine della sperimentazione, si conferma il miglioramento degli esiti nel gruppo sperimentale rispetto al gruppo di controllo in tre misurazioni su sette, nello specifico: controllo inibitorio, memoria di lavoro visuo-spaziale e funzioni ese- cutive combinate. In particolare avanzano quei bambini che, in fase iniziale, hanno riportato punteggi più bassi; questo avvalora la ricaduta positiva di un protocollo di attività, adeguatamente strutturate e caratterizzate da momenti collaborativi, consolidati da domande stimolo che invitano alla riflessione. L'avanzamento nello sviluppo delle funzioni esecutive nel gruppo sperimentale è traducibile in una conquista temporale pari a 6 mesi rispetto al gruppo di controllo.

La memoria di lavoro visuo-spaziale, così come la percezione delle forma e la visualizzazione spaziale, se stimolate e attivate, fungono da predittori della conoscenza matematica in età scolare (Bull et al., 2008; Kyttälä et al., 2003; Zhang \& Lin, 2015). La ricerca condotta da Rittle-Johnson et al. (2019), su un campione di 73 bambini con un'età media di 4 anni e 7 mesi, ha inteso verificare la correlazione esistente tra due importanti fattori alla base della conoscenza matematica: la ripetizione di modelli (modellizzazione) e le abilità spaziali (percezione della forma, visualizzazione spaziale, memoria di lavoro visuo-spaziale). Queste ultime vengono messe in atto tutte le volte in cui i bambini si confrontano con blocchi, puzzle e videogiochi, nei quali viene richiesto di identificare, estrarre o duplicare sche$\mathrm{mi}$ visivi ripetuti per avanzare in sequenze successive. Al termine delle valutazioni effettuate, è stata accertata la correlazione tra le abilità di modellamento e quelle spaziali, contrariamente alla misura delle singole abilità spaziali rispetto alla conoscenza matematica. Ciò nonostante lo studio longitudinale, in linea con le precedenti sperimentazioni, fa emergere la relazione esistente tra la memoria di lavoro visuo-spaziale, intesa quale singola abilità spaziale, e i positivi risultati in prove di matematica riguardanti la numerazione, il calcolo e la geometria ottenuti dai bambini a distanza di due anni dal potenziamento ricevuto. La visualizzazione spaziale gioca un importante ruolo nell'acquisizione della numerazione, infatti sembrerebbe supportare i bambini di scuola primaria nell'elaborazione di strategie aritmetiche più complesse o nella rappresentazione di grandezze numeriche. Di conseguenza, la pratica ripetuta di tali abilità, oltre a sostenere lo sviluppo iniziale del processo di numerazione, incrementa significativamente le prestazioni dei bambini soprattutto se arricchite dai device digitali (Freina \& Bottino, 2019). Pertanto vi è un comune denominatore nei differenti percorsi di ricerca fino a questo punto citati: la presenza di limiti e vincoli che andrebbero colmati. Tra questi, la difficoltà nel monitorare le modalità di intervento attuate in classe per comprendere sino a che punto i programmi intrapresi possano avere delle ricadute positive sugli apprendimenti e la dimensione ridotta del campione che, difficilmente, consente di effettuare 
studi di follow-up per valutazioni più rigorose e a lungo termine.

\section{DISCUSSIONE}

L'analisi e la comparazione delle fonti selezionate hanno consentito di restituire un quadro delle linee di ricerca nel campo dell'intelligenza visuo-spaziale in età prescolare. Dalla breve rassegna emergono delle tendenze ricorrenti che corrispondono alle tre aree tematiche esaminate: (i) memoria di lavoro; (ii) funzioni esecutive; (iii) efficacia dei percorsi di potenziamento cognitivo.

La letteratura nazionale e internazionale restituisce un framework teorico dal quale è desumibile quanto sia importante puntare sull'esercizio delle competenze di base prima della scolarizzazione. La cognizione numerica, ad esempio, necessita di un'appropriata padronanza nel dominio delle abilità sopra menzionate, oltre al fatto che nella fascia d'età 3-5 anni si assiste a una rapidità nel processo di sviluppo e a una forte interdipendenza tra l'ambito motorio, cognitivo e linguistico (Houwen et al., 2016). Inoltre già a partire dai 4 anni la memoria di lavoro visuo-spaziale risulta pienamente correlata con i domini generali relativi al controllo e all'attenzione. Sempre in questi anni, si rafforza anche quel legame tra le funzioni esecutive e le abilità visuo-spaziali, traducibile nel monitoraggio di immagini in sequenza e nelle operazioni di regolazione cognitiva. L'attenzione selettiva, la memoria verbale e visuo-spaziale rappresentano, dunque, dei predittori determinanti nell'apprendimento dei contenuti scolastici poiché rientrano in tutte quelle situazioni didattiche che richiedono il coinvolgono del linguaggio e il ragionamento.

A tal proposito la ricerca evidence based, che ha per oggetto la school readiness e lo scholastic achievement (successo scolastico) (Altinok, 2008; Bull et al., 2008; Clark, 2010; Duncan et al., 2007; Mayer \& Alexander, 2011; Robson \& Quinn, 2017) fornisce un corpus di conoscenze riguardanti i criteri, gli elementi e le condizioni efficaci per promuovere dei percorsi di potenziamento cognitivo che vadano a rinforzare questi aspetti salienti negli anni della scuola dell'infanzia.

Progettare programmi di intervento, sistematicamente organizzati e di intensità crescente, protesi alla stimolazione delle funzioni cognitive di base e ai meccanismi sottesi ai differenti domini di apprendimento, significa facilitare il percorso formativo successivo. $\grave{E}$ questo un periodo particolarmente caratterizzato da un rapido avanzamento del sistema cognitivo e socio-emotivo in cui i fattori contestuali contribuiscono alla costruzione di schemi complessi.
Un ruolo fondamentale è svolto dall'adulto esperto che pone l'allievo in una situazione di apprendimento mediato (Feuerstein et al., 1980; Feuerstein et al., 2018) secondo una prospettiva che mira a considerare la pluralità degli elementi coinvolti nella strutturazione cognitiva. Un allenamento cognitivo ben progettato potrebbe, infatti, favorire la modifica dei circuiti nervosi a seconda degli stimoli sensoriali che provengono dall'ambiente (Green \& Bavelier, 2003). Quanto più ricca è l'esperienza di stimolazione e di mediazione tanto più è garantito il successo dell'intervento formativo. In tale cornice, i giochi computerizzati rappresentano gli strumenti privilegiati per mettere in campo un percorso di potenziamento cognitivo volto a sollecitare le cosiddette core capacities (capacità mentali di base). ${ }^{4}$ La letteratura a riguardo testimonia l'avanzamento delle funzioni cognitive di base mediante l'utilizzo di giochi interattivi, in particolare in tre aree cognitive: attenzione visiva e elaborazione visuo-spaziale; funzioni esecutive e memoria (Bisoglio et al., 2014). I videogiochi d'azione, nei quali si richiede al protagonista di compiere una serie di missioni precedentemente illustrate, modificano in particolar modo l'attenzione selettiva dei novizi dopo un training di stimolazione (Green \& Bavelier, 2006; Wu \& Spence, 2013) e ciò viene verificato anche nell'avanzamento delle prestazioni che richiedono un'elaborazione visuospaziale di livello avanzato (Wu et al., 2012). Trinchero (2014), nel descrivere le potenzialità dei videogiochi computerizzati, coniuga i processi di pensiero di Anderson e Krathwohl (2001) in situazioni di gioco specifiche, esemplificativa è l'operazione del "confrontare" prevista in quelle situazioni nelle quali viene chiesto di trovare le differenze tra figure, mettere in relazione oggetti corrispondenti e così via.

Di conseguenza, per favorire l'autonomia del bambino e incentivare la motivazione e il coinvolgimento nel reiterare azioni deputate all'orientamento, al posizionamento, così come al trascinamento, andrebbero realizzati training di stimolazione più accattivanti ricorrendo ad applicativi digitali touch screen.

I software, ideati a scopo educativo, consentono al bambino di interfacciarsi con più canali comunicativi simultaneamente, così da arricchire le tradizionali esperienze di apprendimento. In tal caso, sono maggiori le occasioni che lo immettono nella condizione di essere al contempo fruitore dei contenuti e autore degli stessi soprattutto quando gli viene richiesto di agire per trasformare, da solo o in maniera collaborativa (Dini \& Ferlino, 2001). Attraverso il videogioco il bambino

\footnotetext{
${ }^{4}$ Le capacità mentali di base riguardano differenti aree quali la percezione, lattenzione, la comprensione, la memoria, il ragionamento e la coordinazione pensiero-azione (Trinchero, 2014).
} 
manipola oggetti concreti che rinviano a concetti astratti. Ad esempio, quando gli viene richiesto di trascinare una figura per completare uno spazio, si sta misurando con il concetto matematico dell'addizione. Il gioco digitale consente inoltre di acquisire le strategie, i criteri e le regole alla base delle soluzioni ai problemi e di sviluppare il ragionamento sin dalla tenera età (Bottino et al., 2007; Trinchero, 2014). Inseriti in attività strutturate, gli applicativi software offrono la possibilità di comprendere le relazioni spaziali, affinando la capacità di discriminare forme e colori, di manipolare gli oggetti, di individuare la posizione che occupano nello spazio. Un'ulteriore capacità che viene promossa è legata alla memorizzazione dei contesti e dei contenuti di diversa natura. Dini e Ferlino (2016), nel riportare una classificazione di app educative, riconoscono l'importanza di tali applicazioni software in quelle attività volte all'avvio dei contenuti propedeutici alla scuola primaria e, a differenza dei software tradizionali che necessitano di una alfabetizzazione informatica pregressa, le app risultano particolarmente congeniali all'interazione autonoma. Come ampiamente descritto in precedenza, sono ben definite le azioni da svolgere per affinare le conoscenze disciplinari tra queste il pensiero matematico, supportato da rappresentazioni spaziali così come da situazioni che richiedono di agire nello spazio, si amplifica attraverso la trasposizione degli oggetti o delle figure per combinarle o separarle e in questo caso svolgere addizioni e sottrazioni (Caviola et al., 2012). Inoltre, le attività ludiche che ricorrono all'uso dei blocchi, se supportate dal docente, migliorano le abilità spaziali proprio in età prescolare.

\section{RIFLESSIONI CONCLUSIVE}

In riferimento all'emergenza Covid-19 e alla sospensione della didattica in presenza, la ricerca in tale area ha evidenziato l'assenza di una riprogettazione didattica in grado di rimodulare gli interventi formativi, adeguando tempi e spazi avvalendosi delle potenzialità offerte dalle tecnologie didattiche (Ranieri et al., 2020).

Per tale ragione, proporre un percorso basato sul potenziamento degli stimoli visivi significa incidere positivamente sulle prestazioni legate al controllo inibitorio (Bodrova \& Leong, 2007) e ciò diviene maggiormente incisivo con giochi computerizzati e interattivi (Mackey et al., 2011).

Le suddette linee di ricerca hanno avvalorato l'intenzione di mettere a punto un modello di software, che è stato implementato in un'applicazione denominata VIEP-app concepita per bambini di età prescolare e finalizzata ad incrementare le loro abilità visuo-spaziali attraverso sessioni ludico-interattive, differenziate per livelli di difficoltà. Questa applicazione è utilizzabile a distanza attraverso un computer, un tablet o uno smartphone collegato ad internet ed offre un programma teso a consolidare quei prerequisiti di base attraverso esercizi incentrati principalmente sul canale visuomotorio. Infatti, in particolar modo quando l'interazione avviene per tramite di uno schermo tattile, il bambino ha la possibilità di trascinare, assemblare, ruotare o manipolare figure e mantiene elevata la sua motivazione grazie ai feedback visivi e sonori forniti da VIEP-App in conseguenza alle sue azioni.

La letteratura esaminata conferma una relazione statisticamente significativa tra le prove di memoria sia verbale che visuo-spaziale ad alto controllo e il pensiero logico. Le abilità visuo-spaziali, sin dalla prima infanzia, sono correlate positivamente con la capacità di conteggio a differenza di quelle verbali. Nello specifico, la memoria di lavoro visuo-spaziale è coinvolta in una varietà di compiti cognitivi che richiedono la generazione, la conservazione e la trasformazione di immagini mentali (Kosslyn, 1980). Riveste, inoltre, un ruolo importante nella comprensione del testo, nel calcolo numerico e nella risoluzione di problemi. In aggiunta viene rimarcata l'incidenza delle funzioni esecutive nella gestione dell'autocontrollo cognitivo e comportamentale, così come nella conservazione delle informazioni rilevanti, inibendo quelle ininfluenti. Per valutare le singole aree che compongono il modello multi-componenziale della memoria di lavoro, le ricerche richiamate individuano una serie di attività, prove e test specifici di seguito sintetizzate da Orsolini (2019). Per l'esecutivo centrale, ad esempio, si fa riferimento ad esercizi volti a rievocare le cifre all'indietro e invertire l'ordine di presentazione delle stesse, in tal modo si misura lo span indiretto o inverso, ossia la capacità della memoria di ritenzione della memoria rispetto agli stimoli ricevuti. Per il loop fonologico la rievocazione di cifre in avanti consente al bambino di rievocare la medesima sequenza, verificando lo span diretto. Per il taccuino visuo-spaziale, ci si avvale del test a blocchi di Corsi che prova il rispetto della sequenza nell'ordine di presentazione dei cubi, in tal modo si misura lo span della memoria visuo-spaziale e l'esecutivo centrale nella riproduzione all'inverso della sequenza dei cubi. Per il buffer episodico ci si può avvalere del Listening Span Test che richiede al bambino di isolare e rievocare le parole conclusive di una serie di frasi, dopo aver accertato la veridicità o falsità delle proposizioni. Si può constatare il vantaggio apportato dai programmi di intervento anche nelle prestazioni di quei bambini che riportano delle difficoltà nell'attivazione delle funzioni esecutive. Ciò si verifica nel momento in cui le attività vengono organiz- 
zate in maniera progressiva con un aumento graduale del livello di complessità rispetto allo stato attuale del bambino. In una situazione come quella della diffusione del COVID-19, in cui il normale svolgimento delle attività didattiche in presenza risulta essere decisamente compromesso, il modello proposto e qui presentato può essere una soluzione su cui riflettere. Essa potrebbe apportare un contributo al ridisegno complessivo dell'azione educativa rivolta a questa delicatissima fascia di età, in quanto consente di affinare specifiche abilità e competenze coinvolgendo i partecipanti nello svolgimento di compiti sfidanti e richiedendo loro di spingersi al di là delle proprie capacità (Bergman Nutley et al., 2011). Il miglioramento delle prestazioni dipende, infatti, sia dal tempo impiegato nell'esercizio di tali abilità che dalla pratica ripetuta, qualora venga inserita in maniera continuativa nei curricula scolastici e non relegata a un periodo circoscritto.

\section{BIBLIOGRAFIA}

Alloway, T. P., Gathercole, S. E., \& Pickering, S. J. (2006). Verbal and visuospatial short-term and working memory in children: Are they separable? Child development, 77(6), 1698-1716. https://doi.org/10.1111/ j.1467-8624.2006.00968.x

Altinok, N. (2008). An International perspective on trends in the quality of learning achievement (1965-2007). UNESCO. https://unesdoc.unesco.org/ark:/48223/ pf0000178009

Amadini, M., Ferrari, S., \& Polenghi, S. (eds.) (2019). Comunità e corresponsabilità educative. Soggetti, compiti e strategie. Pensa multimedia.

Anderson, L. W., \& Krathwohl, D.R. (eds). (2001) A taxonomy for learning, teaching, and assessing. A revision of Bloom's taxonomy of educational objectives. Addison Wesley Longman.

Anderson, P. (2002). Assessment and development of executive function (EF) duringchildhood. Child neuropsychology, 8(2), 71-82. https://doi.org/10.1076/ chin.8.2.71.8724

Anderson, L. M., Shinn, C., Fullilove, M. T., Scrimshaw, S. C., Fielding, J. E., Normand, J., V. G. CarandeKulis \& Task Force on Community Preventive Services. (2003). The effectiveness of early childhood development programs: A systematic review. American journal of preventive medicine, 24(3), 32-46. https:// doi.org/10.1016/S0749-3797(02)00655-4

Anderson, J. R. (2012). Tracking problem solving by multivariate pattern analysis and Hidden Markov Model algorithms. Neuropsychologia, 50(4), 487-498. https:// doi.org/10.1016/j.neuropsychologia.2011.07.025
Baddeley, A. D., \& Hitch, G. J. (1974). Working memory. In G. H. Bower (ed.), The psychology of learning and motivation (pp. 47-89). Academic Press.

Baddeley, A. (1986). Working memory. Oxford University Press.

Baddeley, A. (2000). The episodic buffer: a new component of working memory? Trends in cognitive sciences, 4(11), 417-423. https://doi.org/10.1016/S13646613(00)01538-2

Baddeley, A. D. (2002). Is working memory still working? European Psychologist, 7, 85-97. https://doi. org/10.1027/1016-9040.7.2.85

Bergman Nutley, S., Söderqvist, S., Bryde, S., Thorell, L. B., Humphreys, K., \& Klingberg, T. (2011). Gains in fluid intelligence after training non-verbal reasoning in 4-year-old children: A controlled, randomized study. Developmental science, 14(3), 591-601. https:// doi.org/10.1111/j.1467-7687.2010.01022.x

Bisoglio, J., Michaels, T. I., Mervis, J. E., \& Ashinoff, B. K. (2014). Cognitive enhancement through action video game training: great expectations require greater evidence. Frontiers in Psychology, 5, 136. https://doi. org/10.3389/fpsyg.2014.00136

Bodrova, E., \& Leong, D. J. (2007). Tools of the Mind: The Vygotskian Approach to Early Childhood education. Merrill/Prentice Hall.

Brizzolara, D., Casalini, C., Sbrana, B., Chilosi, A.M., \& Cipriani, P. (1999). Memoria di lavoro fonologica e difficoltà di apprendimento della lingua scritta nei bambini con disturbo specifico di linguaggio. Psicologia Clinica dello Sviluppo, 3, 465-488. https://doi. org/10.1371/journal.pone.0197454

Brocki, K. C., Nyberg, L., Thorell, L. B., \& Bohlin, G. (2007). Early concurrent and longitudinal symptoms of ADHD and ODD: Relations to different types of inhibitory control and working memory. Journal of Child Psychology and Psychiatry, 48(10), 1033- 1041. https://doi.org/10.1111/j.1469-7610.2007.01811.x

Brophy J. (2001). Generic aspects of effective teaching. In M. C. Wang \& H. J. Walberg (eds.), Tomorrow's Teachers (pp. 3-45). McCutchan Publishing Company.

Bruandet, M., Molko, N., Cohen, L., \& Dehaene, S. (2004). A cognitive characterisation of dyscalculia in Turner syndrome. Neuropsychologia, 42(3), 288-298. https:// doi.org/10.1016/j.neuropsychologia.2003.08.007

Bull, R., \& Scerif, G. (2001). Executive functioning as a predictor of children's mathematics ability: Inhibition, switching, and working memory. Developmental neuropsychology, 19(3), 273-293. https://doi. org/10.1207/S15326942DN1903_3

Bull, R., Espy, K. A., \& Wiebe, S. A. (2008). Short-term memory, working memory, and executive func- 
tioning in preschoolers: Longitudinal predictors of mathematical achievement at age 7 years. Developmental Neuropsychology, 33(3), 205-228. https://doi. org/10.1080/87565640801982312

Calvani A. (2007). Evidence-Based Education: ma "funziona" il "che cosa funziona"? Journal of E-Learning and Knowledge Society, 3(3), 139-146.

Calvani A. (2012). Per un'istruzione evidence based. Analisi teorico-metodologica internazionale sulle didattiche efficaci e inclusive. Erickson.

Calvani, A., Zanaboni, B. (2018), Tavole Logico-Visive. Incastri, rotazioni, sviluppi, inferenze, tabelle, punti di vista (3-12 anni). S.Ap.I.E., Società per l'apprendimento e l'Istruzione Informati da Evidenza.

Caviola, S., Mammarella, I. C., Cornoldi, C., \& Lucangeli, D. (2012). The involvement of working memory in children's exact and approximate mental addition. Journal of experimental child psychology, 112(2), 141-160. https://doi.org/10.1016/j.jecp.2012.02.005

Clark, J. M., \& Paivio, A. (1987). A dual coding perspective on encoding processes. In M. A. McDaniel \& M. Pressley (eds.), Imagery and related mnemonic processes (pp. 5-33). Springer.

Clark, R. C., Nguyen, F., Sweller, J. (2006). Efficiency in learning. Evidence-based guidelines to manage cognitive load. Pfeiffer Wiley.

Clark, R. C. (2010). Evidence-Based Training Methods: A Guide for Training Professionals. Astd Press.

Coggi, C., \& Ricchiardi, P. (2014). La «school readiness» e la sua misura: uno strumento di rilevazione per la scuola dell'infanzia. Journal of Educational, Cultural and Psychological Studies (ECPS Journal), 1(9), 283309. https://doi.org/10.7358/ecps-2014-009-cogg

Cornoldi, C., Miato, L., Molin, A., \& Polis, S. (1994). La prevenzione e il trattamento delle difficoltà di lettura e scrittura. Giunti O.S. Organizzazioni Speciali.

Cornoldi, C., Rigoni, F., Tressoldi, P. E., \& Vio, C. (1999). Imagery deficits in nonverbal learning disabilities. Journal of learning disabilities, 32(1), 48-57. https://doi.org/10.1177/002221949903200105

Cornoldi, C., \& Vecchi, T. (2000). Mental Imagery in blind people: The role of passive and active visuospatial processes. In Morton, A. H. (ed.), Touch, representation and blindness (pp. 29- 58). Oxford University Press.

Cornoldi, C., \& Vecchi, T. (2003). Visuo-spatial working memory and individual differences. Psychology Press.

Corsi, P. M. (1972). Human Memory and Medical Temporal Response of the Brain (Doctoral dissertation). McGill University.

Davidson, M.C., Amso, D., Anderson, L.C., \& Diamond, A. (2006). Development of cognitive con- trol and executive functions from 4-13 years: evidence from manipulations of memory, inhibition, and task switching. Neuropsychologia, 44(20), 37-78. https://doi.org/10.1016/j.neuropsychologia.2006.02.006

Dehaene, S., Piazza, M., Pinel, P., \& Cohen, L. (2003). Three parietal circuits for number processing. Cognitive Neuropsychology, 20(3), 487-506. https://doi. org/10.1080/02643290244000239

Del Grande, J. (1990). Spatial sense. Arithmetic Teacher, 37(6), 14-20.

De Ribaupierre, A. D., Lecerf, T., \& Bailleux, C. (2000). Is a non verbal working memory task necessarily nonverbally encoded? Cahiers De Psychologie Cognitive/ Current Psychology of Cognition, 19(2), 135-170. https://archive-ouverte.unige.ch/unige:6792

Diamond, A., Barnett, W.S., Thomas, J., \& Munro, S. (2007). Preschool program improves cognitive control. Science, 318(13), 87-88. https://doi.org/10.1126/ science. 1151148

Diamond, A., \& Lee, K. (2011). Interventions shown to aid executive function development in children 4-12 years old. Science, 333(6045), 959-964. https://doi. org/10.1126/science. 1204529

Diamond, A. (2013). Executive functions. Annual Review of Psychology, 64, 135-168. https://doi.org/10.1146/ annurev-psych-113011-143750

Dini, S., \& Ferlino, L. (2001). Bambini e computer: come cambia il modo di giocare e di imparare. Nuove tecnologie e cambiamenti nell'imparare e nel giocare nei bambini di età prescolare. Italian Journal of Educational Technology, 9(2), 31-41.

Dini, S., \& Ferlino, L. (2016). La conoscenza tra le dita dei bambini. Imparare a giocare a tempo di app. $T D$ Tecnologie Didattiche, 24(3), 147-155. https://www. learntechlib.org/p/183207/

Duff, S. C., \& Logie, R. H. (1999). Storage and processing in visuo-spatial working memory. Scandinavian Journal of Psychology, 40(4), 251-259. https://doi. org/10.1111/1467-9450.404124

Duncan, G. J., Dowsett, C. J., Claessens, A., Magnuson, K., Huston, A. C., Klebanov, P., Pagani, L. S., Feinstein, L., Engel, M., Brooks-Gunn, J., Sexton, H., Duckworth, K., \& Japel, C. (2007). School readiness and later achievement. Developmental Psychology, 43(6), 1428-1446. https://doi.org/10.1037/00121649.43.6.1428

Espy, K. A., McDiarmid, M. M., Cwik, M. F., Stalets, M. M., Hamby, A., \& Senn, T. E. (2004). The contribution of executive functions to emergent mathematic skills in preschool children. Developmental neuropsychology, 26(1), 465-486. https://doi.org/10.1207/ s15326942dn2601_6 
Fabbro, F. (2018). Qualitative syntheses of research in the field of higher education: methodological perspectives, heuristic procedures and practical applications. Form@re-Open Journal per la formazione in rete, 18(1), 137-149. http://dx.doi.org/10.13128/formare- 22567

Fastame, M. C., \& Antonini, R. (2011). Recupero in...abilità visuo-spaziali. Edizioni Erickson.

Feuerstein, R., Rand, Y., Hoffman, M. B., \& Miller, R. (1980). Instrumental Enrichment. An Intervention for Cognitive Modifiability.University Park Press.

Feuerstein, R., Feuerstein, R. S., Falik, L. H., \& Rand, Y. A. (2008). Il programma di arricchimento strumentale di Feuerstein. Fondamenti teorici e applicazioni pratiche. Edizioni Erickson.

Freina, L., \& Bottino, R. (2019). Giochi a supporto dello sviluppo delle abilità visuospaziali. Italian Journal of Educational Technology, 27(2), 91-104. https://doi. org/10.17471/2499-4324/1058

Gagné, R. (1975). Essentials of learning for instruction. Dryden.

Gardner, H. (1983). Frames of mind: The theory of multiple intelligences. Basic Books.

Garon, N., Bryson, S. E., \& Smith, I. M. (2008). Executive function in preschoolers: a review using an integrative framework. Psychological bulletin, 134(1), 31-60. https://doi.org/10.1037/0033-2909.134.1.31

Gathercole, S. E., \& Baddeley, A. D. (1989). Evaluation of the role of phonological STM in the development of vocabulary in children: A longitudinal study. Journal of memory and language, 28(2), 200-213. https://doi. org/10.1016/0749-596X(89)90044-2

Gathercole, S. E., \& Pickering, S. J. (2000). Working memory deficits in children with low achievements in the national curriculum at 7 years of age. British Journal of Educational Psychology, 70(2), 177-194. https://doi.org/10.1348/000709900158047

Gathercole, S. E., Pickering, S. J., Ambridge, B., \& Wearing, H. (2004). The Structure of working memory from 4 to 15 years of age. Developmental Psychology, 40(2), 177-190. https://doi.org/10.1037/00121649.40.2.177

Gathercole, S., \& Alloway, T. P. (2008). Working memory and learning: A practical guide for teachers. Sage.

Gazzetta Ufficiale (2010). LEGGE 8 ottobre 2010, n. 170. Nuove norme in materia di disturbi specifici di apprendimento in ambito scolastico. (10G0192). https://www.aiditalia.org/Media/Documents/legge170_10.pdf

Goff, D. A., Pratt, C., \& Ong, B. (2005). The relations between children's reading comprehension, working memory, language skills and components of reading decoding in a normal sample. Reading and Writing, 18(7-9), 583-616. http://dx.doi.org/10.13128/formare- 22567

Green, C. S., \& Bavelier, D. (2003). Action video game modifies visual selective attention. Nature 423, 534537. https://doi.org/10.1038/nature01647

Green, C. S., \& Bavelier, D. (2006). Effect of action video games on the spatial distribution of visuospatial attention. J. Exp. Psychol. Hum. 32(6), 1465-1478. https://doi.org/10.1037/0096-1523.32.6.1465

Hammill, D. D., Pearson, N. A., \& Voress, J. K. (2003). Test TPV-Percezione visiva e integrazione visuo-motoria. Centro Studi Erickson.

Hawes, Z., Moss, J., Caswell, B., Naqvi, S., \& MacKinnon, S. (2017). Enhancing children's spatial and numerical skills through a dynamic spatial approach to early geometry instruction: Effects of a 32-week intervention. Cognition and Instruction, 35(3), 236264. https://doi.org/10.1080/07370008.2017.1323902

Hitch, G.J., Halliday, S., Schaafstal, A.M., \& Schraagen, M.C. (1988). Visual working memory in young children. Memory and Cognition, 16(2), 120-132. https:// doi.org/10.3758/BF03213479

Holmes, J., Adams, J. W., \& Hamilton, C. J. (2008). The relationship between visuospatial sketchpad capacity and children's mathematical skills. European Journal of Cognitive Psychology, 20(2), 272-289. https://doi. org/10.1080/09541440701612702

Isidori, M. V., \& Prosperi, M. (2019). Lo screening dei prerequisiti dell'apprendimento e il loro potenziamento. Un'indagine nella scuola dell'infanzia nellottica della didattica inclusiva. Italian journal of special education for inclusion, 7(1), 171-188. https:// doi.org/10.7346/sipes-01-2019-14

Kaldenberg, E.R., Watt, S. J. \& Therrien, W. J. (2015). Reading instruction in science for students with learning disabilities. A Meta-Analysis. Learning disability quarterly, 38(3), 160-173. https://doi. org/10.1177/0731948714550204

Kellogg, R. T. (1996). A model of working memory in writing. In C. M. Levy \& S. Ransdell (eds.), The science of writing: Theories, methods, individual differences, and applications (pp. 57-71). Lawrence Erlbaum Associates.

Kellogg, R. T., Whiteford, A. P., Turner, C. E., Cahill, M., \& Mertens, A. (2013). Working memory in written composition: An evaluation of the 1996 model. Journal of Writing Research, 5(2), 159-190.

Kyttälä, M., Aunio, P., Lehto, J. E., Van Luit, J., \& Hautamäki, J. (2003). Visuospatial working memory and early numeracy. Educational and Child Psychology, 20(3), 65-76. 
Knox, H.A. (1913). The differentiation between moronism and ignorance. New York Medical Journal, 98(1913), 564-566.

Kosslyn, S. M., Margolis, J. A., Barrett, A. M., Goldknopf, E. J., \& Daly, P. F. (1990). Age differences in imagery abilities. Child development, 61(4), 995-1010. https:// doi.org/10.1111/j.1467-8624.1990.tb02837.x

Lanfranchi, S., \& Vianello, R. (2009). Caratteristiche della memoria di lavoro in bambini dai 4 ai 6 anni. Giornale italiano di psicologia, 36(3), 613-634. https://doi. org/10.1421/30203

Lauricella, A. R., Blackwell, C. K., \& Wartella, E. (2017). The "new" technology environment: The role of content and context on learning and development from mobile media. In Barr R. \& Linebarger D. (eds.) Media exposure during infancy and early childhood (pp. 1-23). Springer Cham.

Le Corre, M. (2014). Children acquire the later-greater principle after the cardinal principle. British Journal of Developmental Psychology, 32(2), 163-177. https:// doi.org/10.1111/bjdp.12029

Lecerf, T., \& De Ribaupierre, A. (2005). Recognition in a visuospatial memory task: The effect of presentation. European Journal of Cognitive Psychology, 17(1), 47-75. https://doi.org/10.1080/09541440340000420

Logie, R. H. (1986). Visuo-spatial processing in working memory. The Quarterly Journal of Experimental Psychology Section A, 38(2), 229-247. https://doi. org/10.1080/14640748608401596

Logie, R. H., \& Marchetti, C. (1991). Visuo-spatial working memory: Visual, spatial or central executive? In R. H. Logie \& M. Denis (eds.), Advances in psychology (Vol. 80, pp. 105-115). North-Holland.

Logie, R. H. (1995). Visuo-spatial working memory. Psychology Press.

Lowrie, T., Logan, T., \& Ramful, A. (2017). Visuospatial training improves elementary students' mathematics performance. British Journal of Educational Psychology, 87(2), 170-186. https://doi.org/10.1111/bjep.12142

Lucchiari, C. (ed.). (2018). Psicologia a scuola: un percorso pratico-teorico. libreriauniversitaria. it Edizioni.

Maccoby, E. E., \& Jacklin, C. N. (1974). Myth, reality and shades of gray-what we know and dont know about sex differences. Psychology Today, 8(7), 109-112.

Mackey, A. P., Hill, S. S., Stone, S. I., \& Bunge, S. A. (2011). Differential effects of reasoning and speed training in children. Developmental science, 14(3), 582-590. https://doi.org/10.1111/j.1467-7687.2010.01005.x

Maffioletti, S., \& Facchin, A. (eds.). (2016). La visione nellapprendimento del bambino. Indicazioni, prassi e trattamenti: Indicazioni, prassi e trattamenti. FrancoAngeli.
Mayer, R. E., \& Alexander, P. A. (2011). (eds.). Handbook of Research on Learning and Instruction. Routledge.

Mammarella, I. C. (2008). La memoria di lavoro visuospaziale: una rassegna di studi recenti. Giornale italiano di psicologia, 35(3), 509-540. https://www.rivisteweb.it/doi/10.1421/27930

Mammarella, I. C., Lucangeli, D., \& Cornoldi, C. (2010). Spatial working memory and arithmetic deficits in Nonverbal learning difficulties (NLD) children. Journal of Learning Disabilities, 43(5), 455-468. https:// doi.org/10.1177/0022219409355482

Maniscalco, M., Martorana, C., Caci, B., \& Muratore, V. (2015). L'importanza dei prerequisiti e dello screening precoce nella scuola dell'infanzia. International Journal of Developmental and Educational Psychology INFAD Revista de Psicología, 2(1), 219-232. http:// hdl.handle.net/10662/6416

Marotta, L., Trasciani, M., \& Vicari, S. (2008). Test CMF. Valutazione delle competenze metafonologiche. Con $C D-R O M$. Edizioni Erickson.

McKelvie, S. J. (1986). Effects of format of the Vividness of Visual Imagery Questionnaire on content validity, splithalf reliability, and the role of memory in test-retest reliability. British Journal of Psychology, 77(2), 229-236. https://doi.org/10.1111/j.2044-8295.1986.tb01997.x

Miyake, A., \& Shah, P. (eds.). (1999). Models of working memory: Mechanisms of active maintenance and executive control. Cambridge University Press.

Miyake, A., Friedman, N. P., Emerson, M. J., Witzki, A. H., Howerter, A., \& Wager, T. D. (2000). The unity and diversity of executive functions and their contributions to complex "frontal lobe" tasks: A latent variable analysis. Cognitive Psychology, 41(1), 49-100. https://doi.org/10.1006/cogp.1999.0734

Miyake, A., Friedman, N. P., Rettinger, D. A., Shah, P., \& Hegarty, M. (2001). How are visuospatial working memory, executive functioning, and spatial abilities related? A latent-variable analysis. Journal of experimental psychology: General, 130(4), 621-640. https:// doi.org/10.1037/0096-3445.130.4.621

MIUR (1991). Orientamenti dellattività educativa nelle scuole materne statali. https://www.gazzettaufficiale.it/ eli/id/1991/06/15/091A2596/sg

MIUR (2012). Indicazioni nazionali per il curricolo della scuola dellinfanzia e per il primo ciclo diistruzione.http://www.indicazioninazionali.it/wpcontent/ uploads/2018/08/Indicazioni_Annali_Definitivo.pdf

MIUR (2018). Indicazioni nazionali e nuovi scenari https://www.miur.gov.it/documents/20182/0/Indicazioni +nazionali+e+nuovi+scenari/

MIUR (2020). Piano scuola 2020-2021. Documento per la pianificazione delle attività scolastiche, educative 
e formative in tutte le Istituzioni del Sistema nazionale di Istruzione. https://www.miur.gov.it/documents/20182/2467413/Le+linee+guida.pdf/4e4bb4111 f $90-9502-$ f0 1e-d 8841 a 949429 ? version $=1.0$ $\& \mathrm{t}=1593201965918$

Mix, K.S., \& Cheng, Y.-L. (2012). The relation between space and math. In J. B. Benson (ed.), Advances in child development and behavior (pp.197-243). Elsevier

NAEYC (2009). Developmentally Appropriate Practice in Early Childhood Programs Serving Children from Birth through Age 8. Position statement. www.naeyc. org/files/naeyc/ file/positions/PSDAP.pdf

National Early Literacy Panel (2008). Developing Early Literacy: report of the National Early Literacy Panel. Washington, DC: National Institute for Literacy. http://lincs. ed.gov/publications/pdf/NELPreport09. pdf

Newcombe, N.S. (2010). Picture this: Increasing math and science learning by improving spatial thinking. American Educator, 34(2), 29-43.

Noblit, G.W., \& Hare R.D. (1988). Meta-ethnography: Synthesizing qualitative studies. Sage.

Oakhill, J., \& Kyle, F. (2000). The relation between phonological awareness and working memory. Journal of Experimental Child Psychology, 75(2), 152-164. https://doi.org/10.1006/jecp.1999.2529

Orsolini, M., Fanari, R., Serra, G., Cioce, R., Rotondi, A., Dassisti, A., \& Maronato, C. (2003). Primi progressi nell'apprendimento della lettura: una riconsiderazione del ruolo della consapevolezza fonologica. Psicologia clinica dello sviluppo, 7(3), 403-436. https://doi. org/10.1449/11525

Orsolini, M. (Ed.). (2019). Pensando si impara: Stimolare l'attenzione, le funzioni esecutive e la memoria di lavoro nei bambini con bisogni educativi speciali. FrancoAngeli.

Passolunghi, M. C. (2004). Apprendimento matematico: competenza e disabilità nella soluzione dei problemi. Difficoltà in Matematica, 1(1), 27-39.

Passolunghi, M. C., \& Siegel, L. S. (2004). Working memory and access to numerical information in children with disability in mathematics. Journal of experimental child psychology, 88(4), 348-367. https://doi. org/10.1016/j.jecp.2004.04.002

Passolunghi, M.C., \& Mammarella, I.C. (2010). Spatial and visual working memory ability in children with difficulties in arithmetic word problem-solving. European Journal of Cognitive Psychology, 22(6), 944-963. https://doi.org/10.1080/09541440903091127

Passolunghi, M. C., Mammarella, I. C., \& Del Torre, E. (2011). Influenza della memoria di lavoro visiva e spaziale in bambini con difficoltà di apprendimento nella matematica e nella soluzione dei problemi. Psicologia clinica dello sviluppo, 15(3), 573-596. https:// doi.org/10.1449/35888

Pazzaglia, F. (1999). The role of distinct components of visuospatial working memory in the processing of texts. Memory, 7(1), 19-41. https://doi.org/10.1080/741943715

Pennington, B. F., \& Ozonoff, S. (1996). Executive functions and developmental psychopathology. Journal of child psychology and psychiatry, 37(1), 51-87. https:// doi.org/10.1111/j.1469-7610.1996.tb01380.x

Pickering, S. J., Gathercole, S. E., \& Peaker, S. M. (1998). Verbal and visuospatial short-term memory in children: Evidence for common and distinct mechanisms. Memory \& cognition, 26(6), 1117-1130. https://doi.org/10.3758/BF03201189

Ranieri M. (2007). Evidence Based Education: un dibattito in corso. Journal of e-learning and Knowledge Society, 3(3), 147-152.

Ranieri M., Gaggioli C., \& Borges M.K. (2020). La didattica alla prova del Covid-19 in Italia: uno studio sulla Scuola Primaria. Práxis Educativa, 15, 1-20. https:// doi.org/10.5212/PraxEduc.v.15.16307.079

Rimm-Kaufman, S. E., Pianta, R. C., \& Cox, M. J. (2000). Teachers' judgments of problems in the transition to kindergarten. Early childhood research quarterly, 15(2), 147166. https://doi.org/10.1016/S0885-2006(00)00049-1

Rittle-Johnson, B., Zippert, E. L., \& Boice, K. L. (2019). The roles of patterning and spatial skills in early mathematics development. Early Childhood Research Quarterly, 46, 166-178. https://doi.org/10.1016/j. ecresq.2018.03.006

Roberts, R.J., \& Aman, C.J. (1993). Developmental differences in giving directions: Spatial frames of reference and mental rotation. Child Development, 64(4), 1258-1270. https://doi.org/10.1111/j.1467-8624.1993.tb04199.x

Robson, S., \& Quinn, S. F. (eds.). (2014). The Routledge International Handbook of Young Children's Thinking and Understanding. Routledge.

Rotzer, S., Loennekera, T., Kucian, K., Martin, E., Klaver, P., \& Von Aster, M. (2009). Dysfunctional neural network of spatial working memory contributes to developmental dysclalculia. Neuropsychologia, 47(13), 2859-2865. https://doi.org/10.1016/j.neuropsychologia.2009.06.009

Rourke, B. P. (1993). Arithmetic disabilities, specific and otherwise: A neuropsychological perspective. Journal of Learning Disabilities, 26(4), 214-226. https://doi. org/10.1177/002221949302600402

Savelli, E., Franceschi, S., \& Fioravanti, B. (2013). SPEED: Screening Prescolare Età Evolutiva Dislessia. Trento: Erickson. 
Scalisi, T.G., Pelagaggi D., Fanini S., Desimoni M., \& Romano L. (2009). PAC-SI/Prove di abilità cognitive per la scuola dell'infanzia. Edizioni Infantiae.Org, http://www.infantiae.org/pacsi_cons_fono.asp

Scheiman, M., \& Rouse, M. W. (2006). Optometric management of learning-related vision problems. Elsevier Health Sciences.

Sella, F., Berteletti, I., Lucangeli, D., \& Zorzi, M. (2017). Preschool children use space, rather than counting, to infer the numerical magnitude of digits: Evidence for a spatial mapping principle. Cognition, 158, 56-67. https://doi.org/10.1016/j.cognition.2016.10.010

Siegler, R. S., \& Shrager, J. (1984). Strategy choices in addition and subtraction: How do children know what to do. Origins of Cognitive Skills, 1, 229-293.

Terreni, A., Tretti, M. L., \& Corcella, P. R. (2011). IPDA. Questionario osservativo per l'identificazione precoce delle difficoltà di apprendimento. Con CD-ROM. Edizioni Erickson.

Tominey, S. L., \& McClelland, M. M. (2011). Red light, purple light: Findings from a randomized trial using circle time games to improve behavioral self-regulation in preschool. Early Education \& Development, 22(3), 489-519. https://doi.org/10.1080/104092 89.2011 .574258

Trinchero, R. (2009). Gioco? No, imparo! Linee guida per la progettazione e valutazione di software didattico per il potenziamento cognitivo. In C. Coggi (ed.), Potenziamento cognitivo e motivazionale dei bambini in difficoltà. Il Progetto Fenix (pp. 141-173). FrancoAngeli.

Trinchero, R. (2014). Il gioco computerizzato per il potenziamento cognitivo e la promozione del successo scolastico. Un approccio evidence based. Form@re, 3(14), 7-24. https://doi.org/10.13128/formare-15269

Trinchero, R. (2017). Nove concetti chiave per un'istruzione informata dall'evidenza. FORMAZIONE \& INSEGNAMENTO. Rivista internazionale di Scienze dell'educazione e della formazione, 15(2), 113-126. http://dx.doi.org/10.13128/formare-22386

Vecchi, T., \& Cornoldi, C. (1998). Differenze individuali e memoria di lavoro visuo-spaziale. Giornale italiano di psicologia, 25(3), 491-532. http://dx.doi. org/10.1421/207

Verdine, B. N., Golinkoff, R. M., Hirsh-Pasek, K., \& Newcombe, N. (2017). Links between spatial and mathematical skills across the preschool years. Wiley.

Vianello, R., Gini, G., \& Lanfranchi, S. (2015). Psicologia dello sviluppo. UTET università.

Walk, L. M., \& Evers, W. F., Quante, S., \& Hille, K. (2018). Evaluation of a teacher training pro- gram to enhance executive functions in preschool children. PloS one, 13(5) e0197454. https://doi. org/10.1371/journal.pone.0197454

Wechsler, D. (2012). Wechsler Preschool and Primary Scale of Intelligence-Fourth Edition. The Psychological Corporation.

Welsh, M.C., \& Pennington, B.F. (1988). Assessing frontal lobe functioning in children: Views from developmental psychology. Developmental Neuropsychology, 4(3), 199-230. https://doi. org $/ 10.1080 / 87565648809540405$

Wiebe, S. A., Sheffield, T., Nelson, J. M., Clark, C. A., Chevalier, N., \& Espy, K. A. (2011). The structure of executive function in 3-year-olds. Journal of experimental child psychology, 108(3), 436-452. https://doi. org/10.1016/j.jecp.2010.08.008

Wu, S., Cheng, C. K., Feng, J., D’Angelo, L., Alain, C., \& Spence, I. (2012). Playing a first-person shooter video game induces neuroplastic change. J. Cogn. Neurosci. 24(6), 1286-1293. https://doi.org/10.1162/ jocn_a_00192

Wu, S., \& Spence, I. (2013). Playing shooter and driving videogames improves top-down guidance in visual search. Atten. Percept. Psychophys. 75(4), 673-686. https://doi.org/10.3758/s13414-013-0440-2

$\mathrm{Xu}, \mathrm{C}$, \& LeFevre, J. A. (2016). Training young children on sequential relations among numbers and spatial decomposition: Differential transfer to number line and mental transformation tasks. Developmental Psychology, 52(6), 854-866. https://doi.org/10.1037/ dev0000124

Zanetti, M.A., \& Miazza, D. (2003). Test SR 4-5 (School Readiness 4-5 anni) - Prove per l'individuazione delle abilità di base nel passaggio dalla scuola dell'infanzia alla scuola primaria. Erickson.

Zhang, X., \& Lin, D. (2015). Pathways to arithmetic: The role of visual-spatial and language skills in written arithmetic, arithmetic word problems, and nonsymbolic arithmetic. Contemporary Educational Psychology, 4, 188-197. https://doi.org/10.1016/j. cedpsych.2015.01.005 